\title{
Mutational landscape and its clinical significance in paroxysmal nocturnal hemoglobinuria
}

\author{
Fangfei Chen ${ }^{1,2}$, Shimin Hu $\mathbb{E}^{3}$, Jing Ruan ${ }^{1}$, Miao Chen ${ }^{1}$ and Bing Han $\mathbb{D}^{1}$
}

\section{Dear Editor,}

Paroxysmal nocturnal hemoglobinuria (PNH) is an acquired clonal hematopoietic stem cell disorder caused by mutation of the X-linked PIGA gene, resulting in a deficient expression of glycosylphosphatidylinositolanchored proteins, such as CD55 and CD59 ${ }^{1}$. Patients with $\mathrm{PNH}$ may present with hemolytic anemia, thrombosis, and bone marrow failure. The loss of CD55 and CD59 renders PNH erythrocytes susceptible to intravascular hemolysis and thrombosis.

There is a close relationship between $\mathrm{PNH}$ and aplastic anemia (AA). The clinical picture may shift from one to the other during the course of disease ${ }^{2}$. Genes commonly mutated in myeloid neoplasms have been tested in patients with AA, and some carry prognostic significance ${ }^{3}$. For instance, mutations in PIGA, BCOR, and BCORL1 correlate with a better response to immunosuppressive therapy and a longer duration of overall survival and progression-free survival in patients with $\mathrm{AA}$, whereas mutations in $D N M T 3 A, R U N X 1, J A K 2$, JAK3, and CSMD1 are associated with a worse prognosis. However, studies on the mutations of myeloid cancer-related genes in $\mathrm{PNH}$ and on the mechanism of PNH clonal expansion are limited or inconclusive ${ }^{4,5}$. On the other hand, thrombosis is the most common complication in patients with $\mathrm{PNH}^{6,7}$. Although the risk of thrombosis correlates with the $\mathrm{PNH}$ clone size, thrombotic events do occur in patients with small PNH clones. Recent studies have uncovered that mutations rather than PIGA

\footnotetext{
Correspondence: Bing Han (hanbing_li@sina.com)

${ }^{1}$ Department of Hematology, Peking Union Medical College Hospital, Peking Union Medical College and Chinese Academy of Medical Sciences, Beijing, China

2Division of Gastroenterology, The First Affiliated Hospital, Sun Yat-sen University, Guangzhou, China

Full list of author information is available at the end of the article
}

may function as additional risk factors for thrombosis, but the results vary among different studies ${ }^{8,9}$.

In this study, we investigated the mutational profiles of 41 patients with newly diagnosed $\mathrm{PNH}$ as well as the CD59+ and CD59- cell fractions of peripheral blood from $6 \mathrm{PNH}$ patients by whole-exome sequencing. We further examined the relations between these mutations and patients' clinical and laboratory parameters, in particular, we examined the roles of these mutations in the expansion of PNH clones and thrombosis.

The study cohort included 12 patients with PNH and 29 with PNH/AA. There were 29 men and 12 women with a median age of 35 years (range, 15-72). Thirty-nine patients had anemia (Hgb median $78 \mathrm{~g} / \mathrm{L}$, range 36$140 \mathrm{~g} / \mathrm{L}$ ), 20 had leukopenia (WBC median $4.11 \times 10^{9} / \mathrm{L}$, range $\left.1.5-10.9 \times 10^{9} / \mathrm{L}\right)$, and 20 had thrombocytopenia (PLT median $110 \times 10^{9} / \mathrm{L}$, range $11-349 \times 10^{9} / \mathrm{L}$ ). Fifteen patients had pancytopenia. Twenty-three patients had increased unconjugated bilirubin (UCB) and 39 had increased lactate dehydrogenase (LDH). The median PNH clone sizes were 83\% (range, 10-98\%), 82\% (range, $10-98 \%$ ), and $48 \%$ (range, $0-97 \%$ ) by the proportions of FLAER- granulocytes, CD59- granulocytes, and CD59RBCs, respectively.

Thirteen (31.7\%) patients had a history of thrombosis. The median PNH clone size (FLAER negative granulocytes) was $83 \%$ (range, 18-95\%) in those with thrombosis and $83 \%$ (range, $10-98 \%)$ in those without $(\mathrm{p}=0.688)$. Coronary artery was the most common site of thrombosis $(23.1 \%$ of events), followed by visceral vein and/or deep vein (15.4\% of events each). Four patients had thrombosis in multiple sites. Common inherited hypercoagulable states (factor V Leiden mutation, prothrombin gene mutation, deficiency of protein $\mathrm{S}$, protein $\mathrm{C}$, or antithrombin) were not detected in those PNH patients. There was no difference in the baseline characters between patients with or without thrombosis, except the value of D-dimer (Supplementary Table S1). 


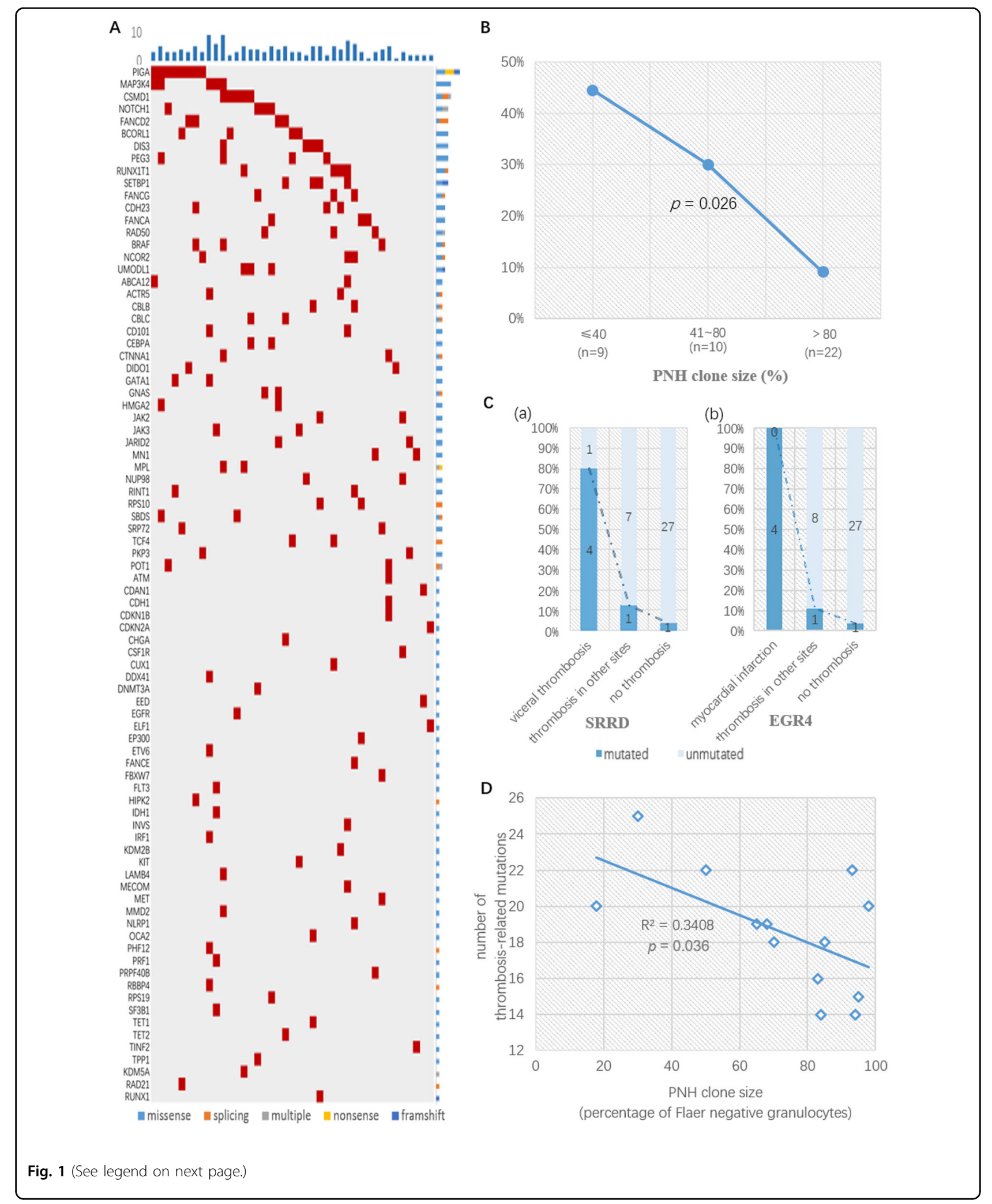

Of the 178 genes frequently mutated in myeloid neoplasms (Supplementary Methods), 158 were mutated in our cohort (Fig. 1A). All 41 patients had mutations and 39 (95\%) had multiple mutations. The average mutation load was 3.85 genes per patient (range, 1-9). As expected, PIGA was most commonly 
(see figure on previous page)

Fig. 1 Mutations in PNH and their clinical correlations. A Heatmap of mutations in myeloid cancer-related genes detected in PNH. One hundred seventy-eight myeloid cancer-related genes were tested by whole-exome sequencing. Every column represented a patient, and every row represented a mutated gene. Red color indicated the detection of mutated gene in this patient. The bar graph above the heatmap showed the number of mutations in each patient. The bar graph on the right side of the heatmap showed the frequency of the type of mutations in each gene. B Negative correlation of PNH clone size with frequency of uncommon mutations in PNH. Patients were divided into different groups $(\leq 40 \%$, $41-80 \%,>80 \%)$ according to their PNH clone size. Genes commonly mutated in and associated with a poor prognosis in aplastic anemia were uncommonly mutated in PNH. These mutated genes were lumped together. The frequency of these mutations declined in PNH as the size of PNH clone increased. C Relationship between gene mutations and sites of thrombosis in PNH. The mutation rates of SRRD and EGR4 in different subgroups of patients were shown in (a) and (b), respectively. The numbers on the columns represented the number of patients in each subgroup. Logistic regression revealed that mutation in SRRD was an independent risk factor for visceral thrombosis $(p=0.032)$ whereas mutation in EGR4 was an independent risk factor for myocardial infarction $(p=0.007)$. D Negative correlation between PNH clone size and frequency of mutations in thrombosis-related genes in PNH. This figure elucidates the PNH clone size calculated by FLAER negative granulocytes as well as the number of mutation in candidate genes concerning thrombosis in 13 patients with thrombosis events. The blue line is the fitted line of the correlation between

mutated and detected in 22 patients (53.7\%). The types of PIGA mutations included truncation $(n=3)$, splicing-site mutations $(n=2)$, frame-shift deletion $(n$ $=2)$, and missense mutation $(n=1)$; additional mutations located in intronic sites $(n=11)$ or in the $3^{\prime}$ untranslated region $(n=3)$. PIGT was mutated in one (2.4\%) patient. Following PIGA gene, the most commonly mutated genes included MAP3K4 and CSMD1, detected in 5 patients $(12.2 \%)$ each. Genes mutated in 4 patients (9.8\%) each included NOTCH1, FANCD2, RUNX1T1, PEG3, DIS3, BCORL1 and SETBP1. Genes mutated in 3 patients (7.3\%) each included FANCG, RAD50, FANCA, CDH23, UMODL1, BRAF, and NCOR2. In addition, 24 genes mutated in 2 patients and 43 in 1 patient each (Supplementary data).

We then examined whether these mutations correlated with clinical and laboratory parameters. We focused on 10 genes with the highest frequency of mutation, including PIGA, BCORL1, RUNXT1, MAP3K, CSMD1, NOTCH1, FANCD2, PEG3, DIS3, and SETBP1 (Table 1). Patents with PIGA mutation had a larger PNH clone size than those without $(90.6 \pm 7.4 \%$ vs $64.4 \pm 32.1 \%, p<0.001)$, and were predominantly females $(58.3 \%$ vs $3.6 \%, p<0.001)$. Patients with BCORL1 mutation were 20 years older $(57 \pm 19$ vs $37 \pm 13$ year-old, $p=0.007)$. Patients with RUNX1T1 mutation had a larger PNH clone of granulocytes (92.8 \pm $5.4 \%$ vs $67.1 \pm 31.3 \%, p<0.001$ ), a lower hemoglobin level $(55.5 \pm 13.3 \mathrm{~g} / \mathrm{L}$ vs $83.7 \pm 22.6 \mathrm{~g} / \mathrm{L}, p=0.020)$, and a higher level of unconjugated bilirubin $(30.9 \pm 16.2 \mu \mathrm{mol} / \mathrm{L}$ vs $15.6 \pm 9.8 \mu \mathrm{mol} / \mathrm{L}, p=0.008)$, suggestive of a higher tendency of hemolysis. No difference in clinical and laboratory parameters was observed between patients with and without 7 other most commonly mutated genes.

Since the incidence of commonly mutated genes associated with a worse outcome in AA was generally lower in PNH patients except for CSMD1 (12.2\%): $2.4 \%$ for $D N M T 3 A, 2.4 \%$ for $R U N X 1,4.9 \%$ for $J A K 2$, and $4.9 \%$ for $J A K 3$, we lumped patients with these mutations together. As one group, patients carrying these mutations had a smaller PNH clone of granulocytes $(48.3 \pm 35.9$ vs $75.5 \pm$
26.8, $p=0.017)$, a lower level of LDH $(731.9 \pm 443.2$ vs 1317.2 $\pm 784.6, p=0.008$ ), and a lower level of UCB $(11.2 \pm 6.7$ vs $18.7 \pm 11.8, p=0.022)$, suggestive of a lower tendency of hemolysis. No difference in other clinical and laboratory parameters was observed between patients with and patients without these uncommonly mutated genes (Supplementary Table S2).

To further explore the correlation between the $\mathrm{PNH}$ clone size and the mutational frequency of genes indicating worse outcome in AA, we divided the patients into 3 groups according to their clone size: $\leq 40 \%(n=9)$, $41-80 \%(n=10)$, and $>80 \%(n=22)$. The mutational frequency of those genes was $44 \%$ (4/9) in patients with PNH clone size $\leq 40 \%, 30 \%(3 / 10)$ in those with clone size of $41-80 \%$, and $9 \%(2 / 22)$ in patients with clone size $>80 \%$. Logistic regression analysis indicated that the mutational frequency declined with the increase of $\mathrm{PNH}$ clone size $(p=0.026)$ (Fig. 1B).

Next we investigated the potential role of mutations in clonal expansion in sorted CD59+ and CD59- cells of peripheral blood from 6 patients with a relatively large $\mathrm{PNH}$ clone. Overall the mutation frequencies were similar in CD59- and CD59+ population, except for PIGA mutation, which was detected only in CD59population. We then searched for those associated with cell proliferation according to the criteria in Supplementary Methods and compared them between sorted CD59- and CD59+ cells. Of the 723 genes associated with cell proliferation, 210 were found mutated in the 6 patients. The mutational loads of those 210 genes were 46.7 (range, 41-53) and 48 (range, 42-52) in the CD59+ and CD59- cells, respectively. Mutated genes unique in CD59- cells included MUC16, NCOR2, PTPN11, CIC, MAML2, BCR, RGPD3, ARID1A, KMT2C, MSH2, NCOR2, and TCL1A, and mutations found in CD59+ cells only included $R O B O 2, S F 3 B 1, H 2 B 3$, and $B C R$. Overall, there was a strong trend toward more cell proliferation or clone expansion associated mutations in CD59- cells than in CD59 + cells $(p=0.062)$ (Supplementary Table S3). 
Table 1 Correlation of common mutations with clinical and laboratory features in PNH.

\begin{tabular}{|c|c|c|c|c|c|c|c|c|c|}
\hline & $\begin{array}{l}\text { Age (year } \\
\text { old, SD) }\end{array}$ & $\begin{array}{l}\text { Sex } \\
\text { (male \%) }\end{array}$ & $\begin{array}{l}\text { Classical } \\
\text { PNH (\%) }\end{array}$ & $\begin{array}{l}\text { Patients with } \\
\text { thrombosis (\%) }\end{array}$ & $\begin{array}{l}\text { FLAER- } \\
(\%, S D)\end{array}$ & $\begin{array}{l}\text { UCB } \\
(\mu \mathrm{mol} / \mathrm{L}, \mathrm{SD})\end{array}$ & $\begin{array}{l}\text { HGB } \\
(g / L, S D)\end{array}$ & $\begin{array}{l}\text { RET } \\
\left(10^{9} / L, S D\right)\end{array}$ & $\begin{array}{l}\text { LDH } \\
(\mathrm{U} / \mathrm{L}, \mathrm{SD})\end{array}$ \\
\hline \multicolumn{10}{|l|}{ PIGA } \\
\hline Mutated & $39(12)$ & $13^{* * *}$ & 50 & 50 & $90.6(7.3)^{* * *}$ & $15.8(10.0)$ & 79.9 (15.1) & $21.0(12.6)$ & 1311 (899) \\
\hline Unmutated & $39(14)$ & $85^{* * *}$ & 45 & 27 & $64.4(32.1)^{* * *}$ & $17.4(11.7)$ & $81.2(25.1)$ & $30.5(63.7)$ & 1159 (734) \\
\hline \multicolumn{10}{|l|}{ MAP3K4 } \\
\hline Mutated & $34(14)$ & 60 & 20 & 20 & $72.4(35.4)$ & $14.2(13.2)$ & $72.8(18.8)$ & $10.8(10.8)$ & 1189 (1057) \\
\hline Unmutated & $40(15)$ & 72 & 50 & 33 & 68.2 (30.6) & $17.5(11.1)$ & 82.1 (23.9) & $30.8(60.8)$ & 1189 (729) \\
\hline \multicolumn{10}{|l|}{ CSMD1 } \\
\hline Mutated & $37(19)$ & 60 & 20 & 0 & $56.6(42.9)$ & $12.6(8.8)$ & $79.8(27.1)$ & $9.9(6.8)$ & 779 (565) \\
\hline Unmutated & $40(14)$ & 72 & 50 & 33 & $71.4(29.0)$ & $17.7(11.5)$ & $81.1(23.2)$ & 31.5 (61.6) & 1246 (771) \\
\hline \multicolumn{10}{|l|}{ NOTCH1 } \\
\hline Mutated & $41(9)$ & 50 & 75 & 50 & $63.0(37.4)$ & $9.8(4.8)$ & $94.5(26.1)$ & $15.0(8.4)$ & 787 (404) \\
\hline Unmutated & $39(15)$ & 73 & 43 & 30 & $70.3(30.5)$ & $17.8(11.5)$ & 79.5 (22.9) & $30.3(61.0)$ & 1232 (779) \\
\hline \multicolumn{10}{|l|}{ FANCD2 } \\
\hline Mutated & $37(16)$ & 20 & 25 & 25 & 67.7 (35.6) & $11.1(8.9)$ & $72.5(20.4)$ & $18.8(19.0)$ & 1165 (1323) \\
\hline Unmutated & $40(14)$ & 76 & 49 & 32 & $69.8(30.7)$ & $17.7(11.4)$ & $81.9(23.7)$ & $29.9(60.9)$ & 1191 (703) \\
\hline \multicolumn{10}{|l|}{ RUNXIT1 } \\
\hline Mutated & $36(4)$ & 100 & 75 & 25 & $92.8(5.4)^{* * *}$ & $30.9(16.2)^{* *}$ & $55.5(13.3)^{*}$ & $31.6(30.4)$ & $1301(565)$ \\
\hline Unmutated & $40(15)$ & 68 & 43 & 32 & $67.0(31.3)^{* * *}$ & $15.6(9.8)^{* *}$ & $83.7(22.6)^{*}$ & $28.4(60.5)$ & 1177 (783) \\
\hline \multicolumn{10}{|l|}{ PEG3 } \\
\hline Mutated & $50(22)$ & 75 & 0 & 25 & $59.5(33.7)$ & $16.7(11.7)$ & $81.3(23.0)$ & $54.6(85.4)$ & 1355 (1141) \\
\hline Unmutated & $38(13)$ & 70 & 51 & 32 & $70.6(30.7)$ & $17.1(11.4)$ & $81.0(23.7)$ & $25.8(54.9)$ & 1171 (727) \\
\hline \multicolumn{10}{|l|}{ DIS3 } \\
\hline Mutated & $40(19)$ & 100 & 75 & 25 & $75.7(15.0)$ & $13.9(6.7)$ & $92.5(34.3)$ & $13.7(10.1)$ & 970 (502) \\
\hline Unmutated & $39(14)$ & 68 & 43 & 32 & $68.9(32.0)$ & $17.4(11.7)$ & 79.7 (22.2) & $30.5(60.9)$ & 1212 (783) \\
\hline \multicolumn{10}{|l|}{ BCORL 1} \\
\hline Mutated & $57(19)^{* *}$ & 50 & 0 & 25 & $53.2(28.6)$ & $17.9(11.1)$ & $65.3(17.3)$ & $53.8(85.4)$ & 1316 (1069) \\
\hline Unmutated & $37(13)^{* *}$ & 73 & 51 & 32 & $71.3(30.8)$ & $17.0(11.4)$ & $82.7(23.5)$ & $25.9(55.0)$ & 1175 (737) \\
\hline \multicolumn{10}{|l|}{ SETBP1 } \\
\hline Mutated & $47(13)$ & 100 & 75 & 0 & $69.0(35.7)$ & $16.9(8.9)$ & $76.3(28.2)$ & $13.1(10.4)$ & 1050 (639) \\
\hline Unmutated & $38(15)$ & 68 & 43 & 35 & $69.6(30.7)$ & $17.1(11.6)$ & 81.5 (23.2) & $30.6(60.9)$ & 1204 (777) \\
\hline
\end{tabular}

Measurement data were presented as average (standard deviation).

FLAER- proportion of fluorescent aerolysin-negative granulocytes, UCB unconjugated bilirubin, $H G B$ hemoglobin, $R E T$ reticulocyte count, $L D H$ lactic dehydrogenase. ${ }^{*} p<0.05,{ }^{* *} p<0.01,{ }^{* * *} p<0.001$.

Then we examined the potential role of mutations in thrombosis. A total of 55 thrombosis-related genes (Supplementary Table S4) were selected as the candidate genes according to the criteria in Supplementary Methods. No difference in mutation rate was found in those candidate genes between CD59+ and CD59- cells. In addition, mutation in SRRD gene was more common in patients with visceral thrombosis than those with thrombosis in other sites ( $80 \%$ vs $12.5 \%, p=0.032$ ), whereas EGR4 mutation was more common in patients with myocardial infarction ( $100 \%$ vs $11.1 \%, p=0.007)$ (Fig. 1C). In patients with thrombosis, the number of those candidate mutations was negatively correlated with the PNH clone size $\left(p=0.036, R^{2}=0.341\right)$ (Fig. 1D), i.e. 
those with relatively smaller $\mathrm{PNH}$ clone sizes tended to have more thrombosis-related mutations, especially mutations of PADI1 ( $p=0.001), \operatorname{SLC} 2 A 9(p=0.002)$ and TCF3 $(p=0.011)$ gene.

In summary, by whole-exome sequencing 10 most frequently mutated genes in PNH included PIGA, BCORL1, RUNX1T1, MAP3K4, CSMD1, NOTCH1, FANCD2, PEG3, DIS3, and SETBP1. PIGA mutation was associated with a larger PNH clone size and female sex, BCORL1 mutation was associated with a younger age, and RUNX1T1 mutation correlated with a larger PNH clone size, a lower hemoglobin level, and a higher level of unconjugated bilirubin. Mutations indicating an unfavorable outcome in AA were uncommon in PNH and as one group associated with a smaller PNH clone size, a lower level of LDH, and a lower level of unconjugated bilirubin. CD59- fraction tended to have more mutations in proliferation-related genes compared with CD59+ fraction. Thrombosis in different sites demonstrated different gene mutations. SRRD mutation was associated with visceral thrombosis and EGR4 mutation was associated with myocardial infarction. For the first time, we demonstrated the clinical significance of mutation profile in $\mathrm{PNH}$, particularly, in $\mathrm{PNH}$ clonal expansion and thrombosis.

\section{Acknowledgements}

This study was supported by grants from Beijing Natural Science Foundation (7192168), the Chinese Academy of Medical Sciences (CAMS) innovation fund for medical sciences (2016-12M-3-004), the National Key Research and Development Program of China (2016YFC0901500) and the Non-profit Central Research Institute Fund of Chinese Academy of Medical Sciences (2019XK 320047).

\section{Author details}

'Department of Hematology, Peking Union Medical College Hospital, Peking Union Medical College and Chinese Academy of Medical Sciences, Beijing,
China. ${ }^{2}$ Division of Gastroenterology, The First Affiliated Hospital, Sun Yat-sen University, Guangzhou, China. ${ }^{3}$ Department of Hematopathology, The University of Texas MD Anderson Cancer Center, Houston, TX, USA

\section{Conflict of interest}

The authors declare no competing interests.

\section{Publisher's note}

Springer Nature remains neutral with regard to jurisdictional claims in published maps and institutional affiliations.

Supplementary information The online version contains supplementary material available at https://doi.org/10.1038/s41408-021-00451-1.

Received: 15 November 2020 Revised: 14 February 2021 Accepted: 18 February 2021

Published online: 16 March 2021

\section{References}

1. Hill, A., DeZern, A. E., Kinoshita, T. \& Brodsky, R. A. Paroxysmal nocturnal haemoglobinuria. Nat. Rev. Dis. Primers. 3, 14 (2017).

2. de Latour, R. P. et al. Paroxysmal nocturnal hemoglobinuria: natural history of disease subcategories. Blood. 112, 3099-3106 (2008).

3. Yoshizato, T. et al. Somatic mutations and clonal hematopoiesis in aplastic anemia. N. Engl. J. Med. 373, 35-47 (2015).

4. Li, L. Y. et al. Deep sequencing of whole genome exon in paroxysmal nocturnal hemoglobinuria. Am J Hematol. 92, E51-E53 (2017).

5. Shen, $\mathbf{W}$. et al. Deep sequencing reveals stepwise mutation acquisition in paroxysmal nocturnal hemoglobinuria. J. Clin. Investig. 124, 4529-4538 (2014)

6. Hill, A., Kelly, R. J. \& Hillmen, P. Thrombosis in paroxysmal nocturnal hemoglobinuria. Blood. 121, 4985-4996 (2013).

7. Hall, C., Richards, S. \& Hillmen, P. Primary prophylaxis with warfarin prevents thrombosis in paroxysmal nocturnal hemoglobinuria (PNH). Blood. 102, 3587-3591 (2003).

8. Li, L. et al. Gene mutations associated with thrombosis detected by wholeexome sequencing in paroxysmal nocturnal hemoglobinuria. Int. J. Lab. Hematol. 41, 424-432 (2019).

9. Long, Z., Du, Y., Li, H. \& Han, B. Polymorphism of the ABO gene associate with thrombosis risk in patients with paroxysmal nocturnal hemoglobinuria. Oncotarget. 8, 92411-92419 (2017). 